\title{
Rare earth interstitials in Ge: A hybrid density functional theory study
}

\author{
E. Igumbor • R. C. Andrew - W. E. Meyer
}

\begin{abstract}
In this work, the results of density functional theory calculations for rare earth (Ce, Pr, Eu and Er) interstitials in Ge are presented. We employed the hybrid functional of Heyd, Scuseria, and Ernzerhof (HSE06) for all the calculations. We calculated the formation energies and charge state transition levels for the tetrahedral $(\mathrm{T})$ and hexagonal $(\mathrm{H})$ configurations of the Ce, Pr, Eu and Er interstitials in Ge. While for the T configuration, the charge states of the Ce and Pr did not induce any thermodynamic accessible transition state level within the band gap of Ge, for both the $\mathrm{T}$ and $\mathrm{H}$ configurations the Eu and Er interstitials in Ge induce deep levels in the band gap. The $\mathrm{H}$ configuration of the Ce interstitial
\end{abstract}

E. Igumbor

Department of Physics, University of Pretoria, Pretoria 0002, South Africa

Department of Mathematical and Physical Sciences, Samuel Adegboyega University, Ogwa

Edo State Nigeria

Tel.: +27-61-7364053

E-mail: elgumuk@gmail.com

R. C. Andrew

Department of Physics, University of Pretoria, Pretoria 0002, South Africa

W. E. Meyer

Department of Physics, University of Pretoria, Pretoria 0002, South Africa

Tel.: +27-12-4202637

Fax: +123-12-3625288

E-mail: wmeyer@up.ac.za 
in Ge induces a shallow donor level at $0.03 \mathrm{eV}$ below the conduction band. The

Eu interstitial exhibits negative- $U$ properties for the $(+2 /-2)$ transition level and the Er interstitial displays characteristics of charge state controlled metastability.

Keywords Defect · rare earth · formation energy · charge state

\section{Introduction}

Over the last few years, rare-earth (RE) doping and defects in materials have been extensively studied because of their potential applications in full colour emission devices $[1,2]$. Study revealed that the intra- $4 f$ shell electronic transitions of the RE ions give rise to sharp emission lines with small energy dispersion [3]. It has been suggested that deep levels defect participate in the energy transfer process from the host to the $\mathrm{RE}^{3+}$ ions [4]. Therefore, the structure of the local environment of $\mathrm{RE}^{3+}$ ions plays a crucial role in the determination of the optical luminescence efficiency of RE doped semiconductors [1]. The size of the RE ion, the number of valence electrons and its electronegativity may differ from the host and thus it may be expected that the RE could induce one or more gap levels occupied by valence electrons [5]. Studies of defects in semiconductor materials have been carried out in the last two decades, in particular defects in $\mathrm{Si}[5]$ and only a few in Ge $[6,2$, $7,8]$ (due to problems with the density functional theory predicting a band gap). While the former has a band gap of $1.17 \mathrm{eV}$ [9], the latter has a narrow band gap of $0.78 \mathrm{eV}[10]$ at $0 \mathrm{~K}$. The narrow band gap of Ge, its high carrier mobility and low voltage have made it a possible alternative to $\mathrm{Si}$ as active layer in advanced electronic devices [11,12]. Impurities influence Ge-based semiconductor devices either positively or negatively [6]. Several experimental and theoretical studies of 
point defects such as vacancy [13] and interstitial $[6,7]$ defects in Ge have been reported in the literature. While $\mathrm{RE}$ ion defects in $\mathrm{Si}$ and other materials have been studied both theoretically and experimentally [14-16], except for Tm $[2,8]$, the structural and electronic properties of RE interstitial defects in Ge have not been experimentally or theoretically reported. In order to provide an insight for the experimental study of the electronic properties of defects resulting from the implantation of RE, we have used the Heyd, Scuseria, and Ernzerhof (HSE06) functional [17] in the framework of density functional theory (DFT) to calculate the electronic properties of the Ce, Pr, Eu and Er interstitials in Ge in two different configurations: the tetrahedral $(\mathrm{T})$ and hexagonal $(\mathrm{H})$. The energies of formation for the $\mathrm{T}$ and $\mathrm{H}$ configurations of the $\mathrm{Ce}, \mathrm{Pr}, \mathrm{Eu}$ and $\mathrm{Er}$ interstitials in Ge were calculated for charge states $(-2,-1,0,+1,+2)$. The charge state transition levels are examined and presented as well. The role of shallow and deep levels, charge state controlled metastability and negative- $U$ properties of charge state transition levels are also discussed. This article is organised as follows: in Section 2, we present the computational details, the result and discussion are presented in Section 3 and finally, we present our concluding remarks in Section 4.

\section{Computational details}

DFT electronic structure calculations were performed using the Vienna ab-initio Simulation Package (VASP) $[18,19]$. We used the Projector-augmented wave (PAW) method, as implemented in the VASP code to separate the inert core electrons from the chemically active valence electrons $[18,20]$. All the calculations were carried out using the Heyd, Scuseria, and Ernzerhof (HSE06) [17] hybrid functional. In 
this approach, the short-range exchange potential is calculated by mixing a fraction $(25 \%)$ of exact nonlocal Hartree-Fock exchange with the generalized gradient approximation (GGA) functional of Perdew, Burke, and Ernzerhof (PBE) [21]. In contrast to the local density approximation (LDA) and the GGA that tend to underestimate the band gap of semiconductors [22,12,23], the HSE06 functional gives an excellent description of the electronic band gap and charge state transition properties for a wide range of the defects in group-IV semiconductors $[22,9$, 7]. For the past decade, the study and prediction of the electronic properties of materials with the $f$ orbital valence shell was difficult due to the fact that the $f$ orbital is highly localized. The highly localized $f$ orbital was previously treated using LDA+U and other methods [24-27]. Recently, the hybrid functional has been successfully used to predict the electronic and band gap properties of several materials with an $f$ orbital in the valence shell $[24,28]$. Following this success of the hybrid functional, it became feasible for us to handle the $f$ orbital in the valence shell of the RE. For Ge, the $4 s$ and $4 p$ electrons in the outer shell were treated as valence electrons, while depending on the electronic configurations, the $6 s, 5 d$, $5 p$ and $4 f$ orbitals in the outer shell were treated as valence electrons for the Ce, Eu, Er and Pr. For the bulk, geometric optimization of Ge was performed using an 8-atoms unit cell with an $8^{3}$ Monkhorst-Pack [29] k-point Brillouin zone sampling scheme and cutoff energy of $400 \mathrm{eV}$. For the defects, we employed a 64 atom supercell using a $2^{3}$ Monkhorst-Pack [29] k-point Brillouin zone sampling scheme, and we set the plane wave cutoff of the wave function expansion to $400 \mathrm{eV}$. We refined the geometry until the change in the total energy was less than $10^{-5} \mathrm{eV}$ and the forces were relaxed to below $0.001 \mathrm{eV} / \AA$. The energy of formation of a system is strongly dependent on the spin-orbit coupling (SOC) due to the presence 
of relativistic effects in heavy atomic systems. To deal with the scalar relativistic effect, the mass-velocity and Darwin correction terms have been incorporated into the PAW potential. In addition to the scalar relativistic effect that was taken into account, spin-orbit coupling was also taken into account for all calculations. The concentrations $(\mathrm{C})$ of defects in thermodynamic equilibrium are related to the formation energy $\left(E^{f}\right)$ through the Boltzmann constant $\left(k_{B}\right)$

$$
C=N_{0} \exp \left(-E^{f} / k_{B} T\right)
$$

where $T$ is temperature in Kelvin and $N_{0}$ is the number of sites in the crystal where the defect can occur per unit volume. For a charged system, the energy of formation of the defect in its charged state depends on the Fermi level $\left(\varepsilon_{F}\right)$. The formation energy $\left(E^{f}\right)$ of a defect is derived directly from total energies calculated by DFT, allowing the calculation of equilibrium defect concentrations [30]. To calculate the defect formation and thermodynamic transition energy $\left(\epsilon\left(q / q^{\prime}\right)\right)$ levels, we calculated the total energy $\mathrm{E}(d, q)$ for a supercell containing the optimized defect $d$ in its charge state $q$. The defect formation energy $E^{f}(d, q)$ as a function of electron Fermi energy $\left(\varepsilon_{F}\right)$ is given as [31]

$$
E^{f}(d, q)=E(d, q)-E(\text { pure })+\sum_{i}(\triangle n)_{i} \mu_{i}+q\left[E_{V}+\varepsilon_{F}\right]+E_{c o r}^{q},
$$

where $E($ pure $)$ is the energy of a supercell without a defect, $(\triangle n)_{i}$ is the difference in the number of constituent atoms of type $i$ between the pristine supercell and the supercell containing the defect, $\mu_{i}$ represents the chemical potential of different constituent atoms and $E_{V}$ is the valence band maximum (VBM). The correction term $E_{c o r}^{q}$ according to Freysoldt et al [32], has been included to account for 
the discrepancies surrounding the calculation of $E^{f}(d, q)$ due to finite-size effects within the supercell and inaccuracy underlying the approximation of the energy due to electrostatic interactions. According to Eq. 1, an increase in the formation energy of a defect obtained from Eq. 2 leads to a decrease in the concentration of a defect, and the defect becomes less energetically favourable. The defect transition energy level $\epsilon\left(q / q^{\prime}\right)$ is the Fermi energy at which two charge states ( $q$ and $q^{\prime}$ ) of the defect have the same energy of formation, and is given as [32]

$$
\epsilon\left(q / q^{\prime}\right)=\frac{E^{f}\left(d, q ; \varepsilon_{F}=0\right)-E^{f}\left(d, q^{\prime} ; \varepsilon_{F}=0\right)}{q^{\prime}-q}
$$

As reported in ref [7], we took the modelled band gap of the pristine Ge to be 0.78 $\mathrm{eV}$.

\section{Results and discussion}

3.1 Structural properties of RE interstitials in Ge

In order to find out the most energetically favourable structure for the neutral charge state of RE interstitials in Ge, two different atomic arrangement were considered: the hexagonal $(\mathrm{H})$ and tetrahedral $(\mathrm{T})$ configurations. The relaxed geometric structures of RE interstitials in Ge are shown in Figs. 1 and 2, for the $\mathrm{T}$ and $\mathrm{H}$ configurations, respectively. Table 1 lists the predicted shortest bond distance $(\AA)$ between RE and Ge atoms, before geometric relaxation $\left(\alpha_{\mathrm{d}}\right)$, after geometric relaxation $\left(\beta_{\mathrm{d}}\right)$ and the difference $\left(\Delta_{\mathrm{d}}\right)$ between $\alpha_{\mathrm{d}}$ and $\beta_{\mathrm{d}}$ for the $\mathrm{T}$ and $\mathrm{H}$ configurations of RE interstitials in Ge. The shortest bond length between $\mathrm{RE}$ and Ge atoms was calculated with respect to the nearest neighbour Ge atom. 
Figs. 1a and $1 \mathrm{~b}$ represent the relaxed geometric structures of the Ce and Pr interstitials in Ge, respectively, for the $\mathrm{T}$ configuration. For this configuration, while Fig. 1c represents the relaxed geometric structure of the Eu interstitial in Ge, Fig. 1d represents the relaxed geometric structure of the Er interstitial in Ge. For the $\mathrm{H}$ configuration, Figs. 2a, 2b and 2c, represent the relaxed geometric structures of the Ce, $\operatorname{Pr}$ and Eu interstitials in Ge, respectively. The relaxed geometric structure of the Er interstitial in Ge for the $\mathrm{H}$ configuration is displayed in Fig. 2d as well. After geometric relaxation, the shortest bond distance between a $\mathrm{RE}$ and its nearest neighbours Ge atoms for both the $\mathrm{T}$ and $\mathrm{H}$ configurations reduced. The bond length between Ce and Ge atoms, reduces by $0.03 \AA$ and $0.06 \AA$, for the $\mathrm{T}$ and $\mathrm{H}$ configurations, respectively. The $\mathrm{Pr}$ and Ge atoms bond length reduced by $0.02 \AA$ in the $\mathrm{T}$ configuration and by $0.03 \AA$ in the $\mathrm{H}$ configuration. For the Eu and Er interstitials in Ge, after structural relaxation, the shortest bond length between Eu and Ge, and Er and Ge atoms reduced by $0.06 \AA$ (for Eu-Ge) and $0.01 \AA$ (for Er-Ge) for the T configuration. For the $\mathrm{H}$ configuration, while the Eu and Ge atoms bond length reduced by $0.08 \AA$ after geometric relaxation, the Er and Ge atoms shortest bond length reduced by $0.03 \AA$. For all RE interstitials in Ge, we found that all the shortest bond lengths between RE and Ge atoms reduced within $0.8 \AA$. We have observed that for all RE interstitials in Ge studied, the RE and Ge shortest bond distance as well as the reduction in bond length after structural relaxation for the $\mathrm{T}$ configuration are lower than that of the $\mathrm{H}$ configuration. This suggests that the RE interstitials in Ge experience more bond length strain in the $\mathrm{H}$ configuration than the $\mathrm{T}$ configuration and should be the key to understanding the difference in the formation energies for both configurations. 
3.2 Projected density of states of RE interstitials in Ge

The plot of electronic properties (spin polarised projected density of states (PDOS)) of RE interstitials in Ge for the $\mathrm{T}$ and $\mathrm{H}$ configurations are shown in Fig. 3. The majority (spin-up) and minority (spin-down) density of states are shown as right and left halves of each plot, respectively. For the pristine Ge, the majority and minority spins are symmetrical for the entire plot (as shown in insert (o) of Fig. 3), suggesting non-spin polarization of the system. RE interstitials in Ge for all defects considered show strong evidence of hybridization between the $s$ and $p$ orbitals of participating atoms. For the Er interstitial in Ge, in the $\mathrm{H}$ configuration, orbital states are densely populated both in the conduction band minimum and valence band maximum (see insert (a) of Fig. 3). But for the T configuration, we observed significant contribution of the orbital states in the conduction band minimum, below the Fermi level (see insert (b) of Fig. 3). Nevertheless, in contrast to the T configuration which did not show any full metallic behaviour, the PDOS of the $\mathrm{H}$ configuration of the Er interstitial in Ge exhibits metallic character. For the Eu interstitial (insert (c) and (d) of Fig. 3), the defect induced orbital states in the band gap for both the $\mathrm{T}$ and $\mathrm{H}$ configurations, resulting in 0 band width. This suggests that as a result of the defect introduced, the entire system becomes metallic. For the Ce interstitial in Ge, apart from the hybridization that occurred between the $s$ and $p$ orbitals, the band width of the T configuration is higher than that of the $\mathrm{H}$ configuration. More orbital states are found below the Fermi level at the conduction band minimum for the $\mathrm{T}$ than that of the $\mathrm{H}$ configuration as shown in insert (f) and (e), respectively of Fig. 3. This same trend is observed for the Pr interstitial in Ge. where the difference between the band width of the T 
and $\mathrm{H}$ configurations is $0.37 \mathrm{eV}$. Except for the Pr, all other RE interstitial in Ge studied are spin polarised. Fig. 3 shows that some RE interstitials in Ge introduced states within the band gap of Ge. Depending on the RE, RE interstitials in Ge induced orbital states appear to be either deep (in the middle of the band gap of Ge) or shallow (appearing within the band gap but close to the either conduction band minimum or valence band maximum). It is noted that apart from $\mathrm{Eu}, \mathrm{RE}$ interstitials in Ge for the $\mathrm{T}$ configuration induces less orbital states in the band gap of Ge than the $\mathrm{H}$ configuration. This could play a major role in predicting the sequence of the formation energy of RE interstitials in Ge.

In order to understand the bonding characteristic and charge transfer (relative to host atoms) for the $\mathrm{T}$ configuration of $\mathrm{RE}$ interstitials in $\mathrm{Ge}$, we performed total charge density calculation. Table 2 shows the charge transfer between a RE interstitial atom relative to host atoms. Our results show that there is charge transfer between a participating $\mathrm{RE}$ and Ge atoms. The $\operatorname{Pr}$ and Ce have the least charge transfer of 1.04 and $1.05 \mathrm{eV}$ with Ge. The Eu on the other hand, has the highest charge transfer of $1.42 \mathrm{eV}$ with Ge atom compared to the other participating RE.

3.3 Formation energies of RE interstitials in Ge

The results of the formation energies of the Ce, Pr, Eu and Er interstitials in Ge are tabulated in Table 3. The formation energies for the $\mathrm{T}$ and $\mathrm{H}$ configurations of the Ce interstitial in Ge increase from +2 to the -2 charge state. The energies of formation of the Ce interstitial in Ge for both the $\mathrm{T}$ and $\mathrm{H}$ configurations are between -4.45 and $2.61 \mathrm{eV}$ for all charge state. In addition, the $\mathrm{T}$ configuration 
have lower formation energies for all the charge states than the $\mathrm{H}$ configuration. This suggests that under equilibrium conditions the $\mathrm{T}$ configuration is more energetically favourable in all charge states than the $\mathrm{H}$ configuration.

The Er interstitial in Ge forms with formation energies between 0.28 and $7.64 \mathrm{eV}$ for all charge states in the $\mathrm{T}$ and $\mathrm{H}$ configurations. While the formation energy of the $\mathrm{H}$ configuration tends to increased from the -2 to the +2 charge state, the formation energies for the T configuration decreased. Charged state controlled metastable defects are important in the study of semiconductor. Charged state controlled metastability suggests that even though two different configurations of the same defect type have the same number and type of atoms, the stability of one configuration over the other is charge state dependent $[33,34]$. For the Er interstitial in Ge, the minimum energy configuration of the defect depends on its charge state, suggesting that the defect may display charge state controlled metastability. The formation energies of both the $\mathrm{T}$ and $\mathrm{H}$ configurations for the $\mathrm{Eu}$ interstitial in Ge show an increasing tendency from the +2 charge state to the -2 charge state. While for both the $\mathrm{T}$ and $\mathrm{H}$ configurations of the Eu interstitial in Ge, the formation energies are between 3.74 and $7.82 \mathrm{eV}$, the $\mathrm{T}$ configuration has a lower formation energies than the $\mathrm{H}$ configuration. The difference between the formation energy of the $\mathrm{T}$ and $\mathrm{H}$ configuration for the $0,-1+1-2$ and +2 charge states is greater than $1.5 \mathrm{eV}$ for all charge state. In addition to the Ce, the Eu and Pr interstitials in Ge are energetically more favourable in the $\mathrm{T}$ configuration than the $\mathrm{H}$ configuration. For the Pr interstitial in Ge, the $\mathrm{T}$ configuration has lower formation energies than the $\mathrm{H}$ configuration. Except for the formation energies of the Eu and Er interstitials in Ge which are reasonably high (compared to other RE in this study) for some charge states, the Ce and Pr interstitials in Ge have 
the lowest formation energies in all the configurations.

The Eu interstitial in Ge has the highest formation energies in both the $\mathrm{T}$ and $\mathrm{H}$ configuration for charge state $-2,-1$ and 0 . While the Er interstitial has the highest formation energies for charge state +1 and +2 for the $\mathrm{H}$ configuration, the $\mathrm{Eu}$ interstitial in Ge forms with the highest formation energy for +1 and +2 charges states for the $\mathrm{T}$ configuration. For the $\mathrm{T}$ and $\mathrm{H}$ configurations of RE interstitials in Ge, in the neutral charge state, the formation energy increases in the sequence $\operatorname{Pr}<\mathrm{Ce}<\operatorname{Er}<\mathrm{Eu}$. One of the possible reasons for such a sequence could be attributed to the difference in charge transfer between a RE atom and Ge. While Pr interstitial in Ge has the lowest formation energy for the neutral and negative charge states, the Ce interstitial in Ge has the lowest formation energies for the positive charge states for all configurations. Our calculated formation energies indicate that except for the Er, RE interstitials in Ge under equilibrium conditions are more energetically favourable in the $\mathrm{T}$ than the $\mathrm{H}$ configuration. This could be as a result of the less strain experience by the RE-Ge atoms bond lengths in the $\mathrm{T}$ configuration. Another probable reason why there are differences in formation energies, is as a result of the effect of orbital hybridization between a RE atom and its nearest Ge neighbour as seen in the plot of the PDOS.

3.4 Charge state transition levels of RE interstitials in Ge

Table 4 shows the calculated energies of the charge state thermodynamic transition levels in the band gap of Ge. Plots of the formation energy as a function of the Fermi energy are shown in Fig. 4. For the Ce interstitial in Ge (see Fig. 4a), there is no evidence of charge state transition level within the band gap for the $\mathrm{T}$ 
configuration. The +2 charge state is stable for all $\varepsilon_{F}$ in the band gap. For the $\mathrm{H}$ configuration of Ce interstitial in Ge, we found a transition level at $(+2 /+1)$, which is a shallow donor lying close to the conduction band minimum (CBM) at $E_{C}-0.03 \mathrm{eV}\left(E_{C}\right.$ is the energy of the conduction band minimum). This suggests that the energetic distance to the band edge is within a few $k_{B} T$ at room temperature, resulting in efficient ionization of electrons from the defect level into the conduction band (leading to mobile electrons).

For the Pr interstitial in Ge, there is no evidence of charge state transition level found within the band gap for both the $\mathrm{T}$ and $\mathrm{H}$ configurations. For the $\mathrm{H}$ configuration of $\operatorname{Pr}$ interstitial in Ge, the -1 charge state is stable for all $\varepsilon_{F}$ in the band gap. For the T configuration of the Pr interstitial in Ge, the neutral charge state is stable for all $\varepsilon_{F}$ in the band gap as shown in Fig. 4b. According to Fig. 4c, for the $\mathrm{Eu}$ interstitial in $\mathrm{Ge}$, the $\mathrm{H}$ configuration is predicted to induce deep and shallow levels within the band gap. These levels are deep at $E_{V}+0.26$ and $E_{C}-0.26 \mathrm{eV}$ for the double donor $(+2 /+1)$ and $(+1 / 0)$, respectively and reasonably shallow lying close to the CBM at $E_{C}-0.14 \mathrm{eV}$ for the single acceptor $(0 /-1)$. Other levels found in this configuration are not thermodynamically accessible. For the T configuration of the Eu interstitial in Ge, while there is neither a donor nor acceptor level found, we found an interesting charge state transition level at $(+2 /-2)$, which is lying deep in the band gap at $E_{V}+0.18 \mathrm{eV}$. The interaction energy between two electrons in a two-level defect is referred to as Hubbard U [23,35]. A defect often has a negative- $U(U<0)$ if the atomic position of the defect depends sensitively on its charge state. The Eu interstitial in Ge displays negative- $U$ ordering at $(+2 /-2)$, i.e charged state $+1,0$ and -1 are unstable with respect to dissociating into $q=+2$ and $q=-2$. We calculated the effective negative- $U$ of 
the Eu interstitial in Ge for the T configuration to be $-1.50 \mathrm{eV}$.

Fig. 4d indicates the presence of a deep level created by Er interstitial in Ge, for both the $\mathrm{T}$ and $\mathrm{H}$ configurations. For the $\mathrm{T}$ configuration, the Er interstitial induces a transition state at $(+2 /+1)$ which is a deep donor level lying at $E_{C}-0.28 \mathrm{eV}$. For the $\mathrm{H}$ configuration, instead of observing a donor level, we found a (-1/-2) acceptor level lying far away from the valence band maximum at $E_{C}-0.18 \mathrm{eV}$. According to Fig. 5, for the T configuration, only the Er and $\mathrm{Eu}$ interstitials induce charge state transition levels within the band gap of Ge. For the $\mathrm{H}$ configuration of $\mathrm{RE}$ interstitials in Ge, all except Pr interstitial induce charge state transition levels within the band gap of Ge.

\section{Summary}

In conclusion, results of the tetrahedral $(\mathrm{T})$ and the hexagonal $(\mathrm{H})$ configurations of the $\mathrm{Ce}, \mathrm{Pr}, \mathrm{Eu}$ and Er interstitials in Ge are presented. For all calculations, the Heyd, Scuseria, and Ernzerhof (HSE06) hybrid functional was used in the framework of density functional theory (DFT). The formation energies and charge state transition levels for Re interstitials in Ge are described in detail. We have shown that the formation of $\mathrm{Ce}, \mathrm{Pr}, \mathrm{Eu}$ and Er interstitials in Ge exist with formation energies between -4.76 and $7.82 \mathrm{eV}$ for all charge states, with that of Eu and Er interstitials in Ge slightly higher for some charge states. Our calculations show that of all the four elements, the Ce and Pr interstitials in Ge, have the lowest formation energies in both the $\mathrm{T}$ and $\mathrm{H}$ configurations. For the $\mathrm{Ce}, \mathrm{Pr}$, and $\mathrm{Eu}$ interstitials in Ge, the formation energies for the $\mathrm{T}$ configuration are lower than the $\mathrm{H}$ configurations in all charge states. The Er interstitial in Ge exhibits 
properties of charge state controlled metastability.

We have shown that unlike the Pr interstitial in Ge, that has no induced accessible transition level within the band gap, the Er and Eu interstitials in Ge induce charge state transition levels in the band gap. While the $\mathrm{H}$ configuration of Eu interstitial induces double and single donor levels at $E_{V}+0.26$ and $E_{C}-0.26 \mathrm{eV}$ respectively, the $\mathrm{T}$ configuration of the Eu interstitial in Ge displays negative- $U$ ordering at $(+2 /-2)$ transition level. The Er interstitial in Ge induces a donor level in the $\mathrm{T}$ configuration and an acceptor level in the $\mathrm{H}$ configuration at $E_{C}-0.28$ and $E_{C}-0.18 \mathrm{eV}$ respectively.

\section{Acknowledgement}

This work is based on the research supported partly by National Research foundation (NRF) of South Africa (Grant specific unique reference number (UID) 98961). The opinions, findings and conclusion expressed are those of the authors and the NRF accepts no liability whatsoever in this regard.

\section{References}

1. S. Hernández, R. Cuscó, L. Artús, E. Nogales, R. Martin, K. ODonnell, G. Halambalakis, O. Briot, K. Lorenz, E. Alves, Optical Materials 28(6), 771 (2006)

2. E. Igumbor, W. Meyer, Materials Science in Semiconductor Processing 43, 129 (2016)

3. A. Colder, P. Marie, T. Wojtowicz, P. Ruterana, S. Eimer, L. Méchin, K. Lorenz, U. Wahl, E. Alves, V. Matias, et al., Superlattices and Microstructures 36(4), 713 (2004)

4. C.W. Lee, H.O. Everitt, D. Lee, A. Steckl, J. Zavada, Journal of applied physics 95(12), $7717(2004)$

5. R. Jones, Optical Materials 28(6), 718 (2006)

6. C. Claeys, E. Simoen, 2nd edn. (Elsevier, 2011) 
7. E. Igumbor, C. Ouma, G. Webb, W. Meyer, Physica B: Condensed Matter 480, 191 (2016)

8. E. Igumbor, R.E. Mapasha, R. Andrew, W.E. Meyer, Computational Condensed Matter 8, $31(2016)$

9. P. Deák, B. Aradi, T. Frauenheim, E. Janzén, A. Gali, Phys. Rev. B 81, 153203 (2010)

10. F.J. Morin, J.P. Maita, Phys. Rev. 94, 1525 (1954)

11. L. Lee, E.A. Fitzgerald, T. Bulsara, Mayank, T. Currie, A. Lochtefeld, Journal of Applied Physics 97(1), 011101 (2005)

12. P. Śpiewak, J. Vanhellemont, K. Sueoka, K. Kurzydłowski, I. Romandic, Materials Science in Semiconductor Processing 11(5), 328 (2008)

13. S. Coelho, F. Auret, P.J. van Rensburg, J. Nel, Physica B: Condensed Matter 439(0), 97 (2014)

14. G.S. Pomrenke, E. Silkowski, J.E. Colon, D.J. Topp, Y.K. Yeo, R.L. Hengehold, Journal of Applied Physics 71(4) (1992)

15. Y. Miyata, Y. Nose, T. Yoshimura, A. Ashida, N. Fujimura, Journal of Crystal Growth 425, $158(2015)$

16. H. Przybylinska, W. Jantsch, Y. Suprun-Belevitch, M. Stepikhova, L. Palmetshofer, G. Hendorfer, A. Kozanecki, R.J. Wilson, B.J. Sealy, Phys. Rev. B 54, 2532 (1996)

17. J. Heyd, G.E. Scuseria, M. Ernzerhof, The Journal of Chemical Physics 118(18), 8207 (2003)

18. G. Kresse, J. Furthmüller, Phys. Rev. B 54, 11169 (1996)

19. G. Kresse, D. Joubert, Phys. Rev. B 59, 1758 (1999)

20. P.E. Blochl, Phys. Rev. B 50, 17953 (1994)

21. J.P. Perdew, K. Burke, M. Ernzerhof, Phys. Rev. Lett. 77, 3865 (1996)

22. H. Tahini, A. Chroneos, R.W. Grimes, U. Schwingenschlgl, H. Bracht, Applied Physics Letters 99(7), $072112(2011)$

23. E. Igumbor, K. Obodo, W.E. Meyer, Solid State Phenomena 242, 440 (2015)

24. B.G. Janesko, T.M. Henderson, G.E. Scuseria, Physical Chemistry Chemical Physics $\mathbf{1 1}(3), 443(2009)$

25. V.I. Anisimov, J. Zaanen, O.K. Andersen, Physical Review B 44(3), 943 (1991)

26. L. Petit, A. Svane, Z. Szotek, W.M. Temmerman, Physical Review B 72(20), 205118 (2005)

27. M. Cococcioni, S. De Gironcoli, Physical Review B 71(3), 035105 (2005) 
28. J.L. Da Silva, M.V. Ganduglia-Pirovano, J. Sauer, V. Bayer, G. Kresse, Physical Review B 75(4), $045121(2007)$

29. H.J. Monkhorst, J.D. Pack, Phys. Rev. B 13, 5188 (1976)

30. S.B. Zhang, J.E. Northrup, Phys. Rev. Lett. 67, 2339 (1991)

31. Y. Kumagai, F. Oba, Phys. Rev. B 89, 195205 (2014)

32. C. Freysoldt, J. Neugebauer, C.G. Van de Walle, physica status solidi (b) 248(5), 1067 (2011)

33. M. Risse, R. Vianden, Journal of applied physics 93(5), 2648 (2003)

34. J. Dkabrowski, M. Scheffler, Materials Science Forum 83, 735 (1992)

35. Freysoldt, Christoph, Grabowski, Blazej, Hickel, Tilmann, J. Neugebauer, Kresse, Georg, Janotti, Anderson, V. de Walle, C. G., Rev. Mod. Phys. 86, 253 (2014)

Table 1 Predicted shortest bond distance $(\AA)$ between RE and Ge atoms, before geometric relaxation $\left(\alpha_{\mathrm{d}}\right)$, after geometric relaxation $\left(\beta_{\mathrm{d}}\right)$ and the difference $\left(\Delta_{\mathrm{d}}\right)$ between $\alpha_{\mathrm{d}}$ and $\beta_{\mathrm{d}}$ for the $\mathrm{T}$ and $\mathrm{H}$ configurations of RE interstitials in Ge. The bond lengths are calculated with respect to the nearest neighbour Ge atoms around the RE.

\begin{tabular}{llll|lll}
\hline RE-Ge & \multicolumn{3}{c|}{$\mathrm{T}$} & \multicolumn{3}{c}{$\mathrm{H}$} \\
\hline & $\alpha_{\mathrm{d}}$ & $\beta_{\mathrm{d}}$ & $\Delta_{\mathrm{d}}$ & $\alpha_{\mathrm{d}}$ & $\beta_{\mathrm{d}}$ & $\Delta_{\mathrm{d}}$ \\
\hline $\mathrm{Ce}-\mathrm{Ge}$ & 2.67 & 2.64 & 0.03 & 2.83 & 2.77 & 0.06 \\
$\mathrm{Pr}-\mathrm{Ge}$ & 2.31 & 2.29 & 0.02 & 2.77 & 2.74 & 0.03 \\
$\mathrm{Eu}-\mathrm{Ge}$ & 2.77 & 2.71 & 0.06 & 2.95 & 2.87 & 0.08 \\
$\mathrm{Er}-\mathrm{Ge}$ & 2.67 & 2.66 & 0.01 & 2.70 & 2.67 & 0.03 \\
\hline
\end{tabular}

Table 2 Calculated the total charge $(\mathrm{eV})$ transfer between a $\mathrm{RE}$ and Ge atoms, for the $\mathrm{T}$ configuration of RE interstitials in Ge.

\begin{tabular}{ccccc}
\hline Defect & Pr & Ce & Er & Eu \\
\hline Charge transfer & 1.04 & 1.05 & 1.21 & 1.42 \\
\hline
\end{tabular}


Table 3 Calculated formation energies $\left(E^{f}\right)$ in $\mathrm{eV}$ at $\epsilon_{f}=0$ of the $\mathrm{Ce}$, Eu Er and $\operatorname{Pr}$, interstitials in Ge. The configuration with the lowest formation energies for each charge state are written in bold.

\begin{tabular}{ccccccc}
\hline \multirow{2}{*}{ Defect } & Configuration & -2 & -1 & 0 & +1 & +2 \\
\hline \multirow{2}{*}{$\mathrm{Ce}$} & $\mathrm{T}$ & $\mathbf{- 0 . 3 4}$ & $\mathbf{- 1 . 6 2}$ & $\mathbf{- 2 . 7 4}$ & $\mathbf{- 3 . 6 9}$ & $\mathbf{- 4 . 4 5}$ \\
& $\mathrm{H}$ & 2.61 & 0.99 & 0.04 & -0.87 & -1.55 \\
\hline $\mathrm{Er}$ & $\mathrm{T}$ & 3.83 & 2.65 & $\mathbf{1 . 6 1}$ & $\mathbf{0 . 7 8}$ & $\mathbf{0 . 2 8}$ \\
& $\mathrm{H}$ & $\mathbf{2 . 0 6}$ & $\mathbf{1 . 4 0}$ & 4.51 & 7.64 & 7.21 \\
\hline $\mathrm{Eu}$ & $\mathrm{T}$ & $\mathbf{4 . 4 9}$ & $\mathbf{5 . 3 0}$ & $\mathbf{4 . 6 1}$ & $\mathbf{4 . 0 3}$ & $\mathbf{3 . 7 4}$ \\
& $\mathrm{H}$ & 7.82 & 6.80 & 6.17 & 5.64 & 5.39 \\
\hline $\mathrm{Pr}$ & $\mathrm{T}$ & $\mathbf{- 2 . 6 1}$ & $\mathbf{- 3 . 8 1}$ & $\mathbf{- 4 . 7 6}$ & $\mathbf{- 1 . 6 0}$ & $\mathbf{- 2 . 4 0}$ \\
& $\mathrm{H}$ & 1.35 & -0.43 & -0.12 & 1.89 & 1.24 \\
\hline
\end{tabular}

Table 4 The energy of the charge state transition levels $\epsilon\left(q / q^{\prime}\right)$ in the band gap for the Ce, $\mathrm{Eu}$ Er and Pr, interstitials in Ge for both the $\mathrm{T}$ and $\mathrm{H}$ configurations. These transition levels are taken with reference to the VBM.

\begin{tabular}{llll|llll}
\hline Charge state transition level & \multicolumn{3}{c|}{$\mathrm{T}$} & \multicolumn{5}{c}{$\mathrm{H}$} \\
\hline & $\mathrm{Ce}$ & $\mathrm{Er}$ & $\mathrm{Eu}$ & $\mathrm{Ce}$ & $\mathrm{Er}$ & $\mathrm{Eu}$ \\
\hline$(+2 /+1)$ & - & 0.50 & - & 0.75 & - & 0.26 \\
$(+1 / 0)$ & - & - & - & - & - & 0.52 \\
$(0 /-1)$ & - & - & - & - & - & 0.64 \\
$(-1 /-2)$ & - & - & - & - & 0.60 & - \\
$(+2 /-2)$ & - & - & 0.18 & - & - & - \\
\hline
\end{tabular}




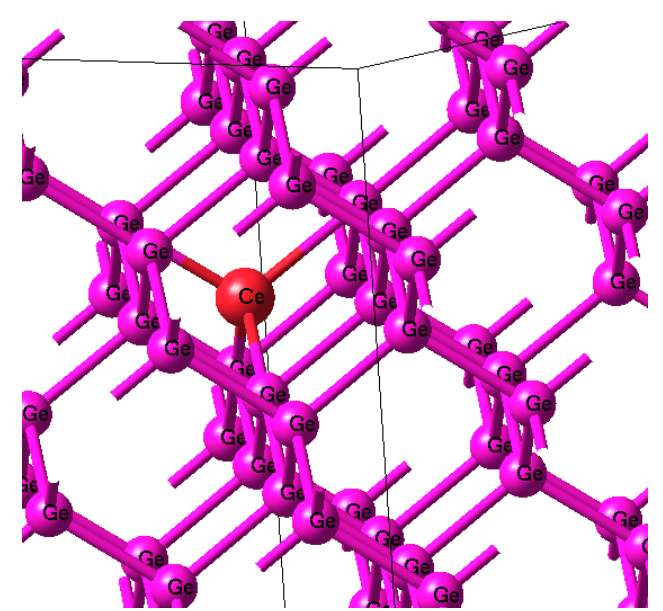

(a)

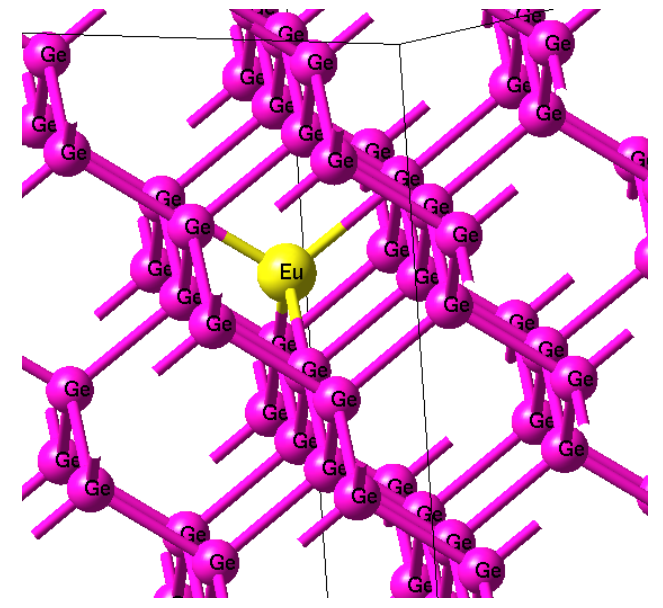

(c)

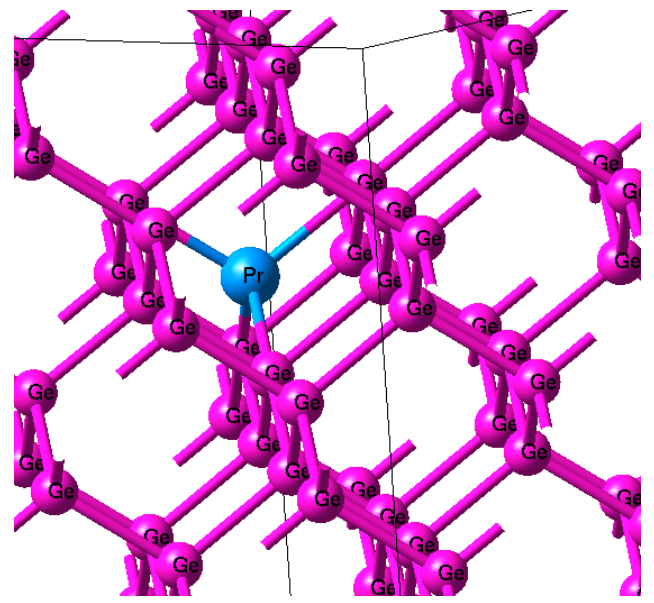

(b)

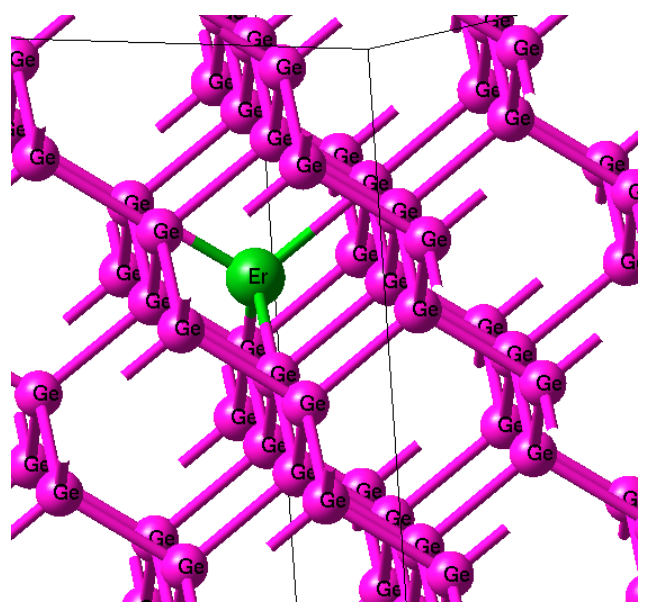

(d)

Fig. 1 Relaxed geometric structures for the T configuration of RE interstitials in Ge. (a) Ce, (b) Pr, (c) Eu and (d) Er. 


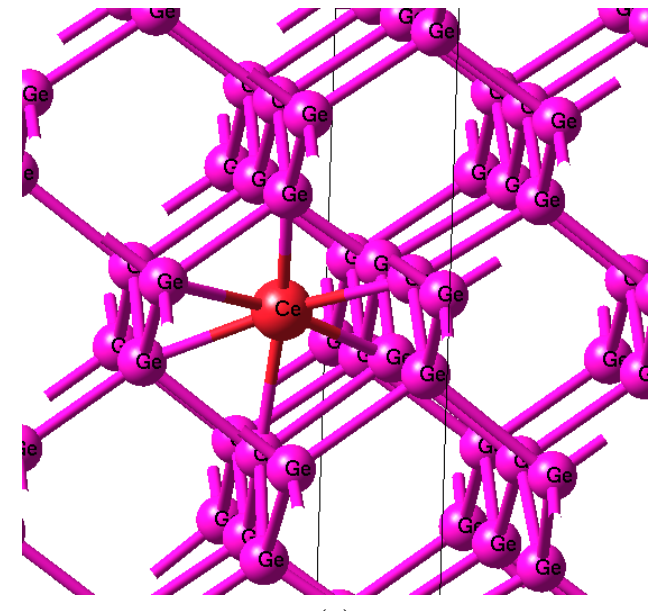

(a)

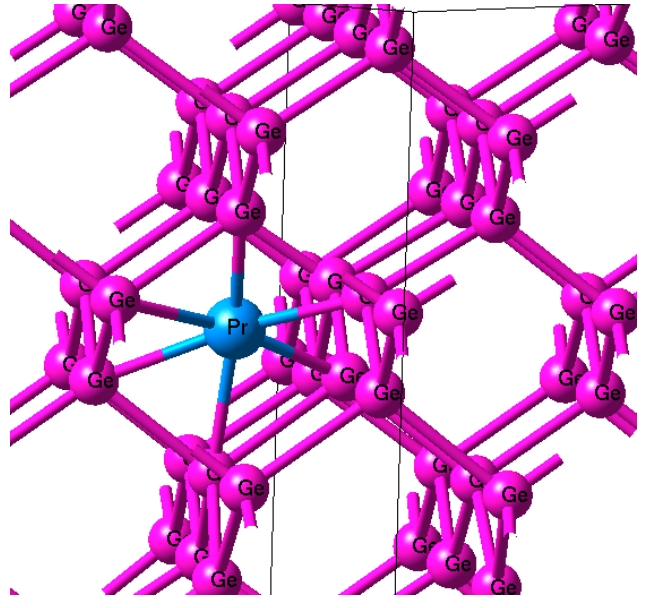

(b)

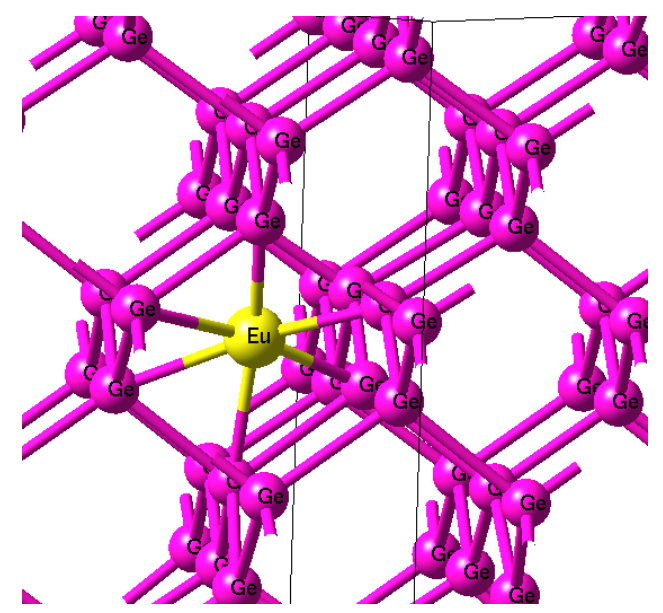

(c)

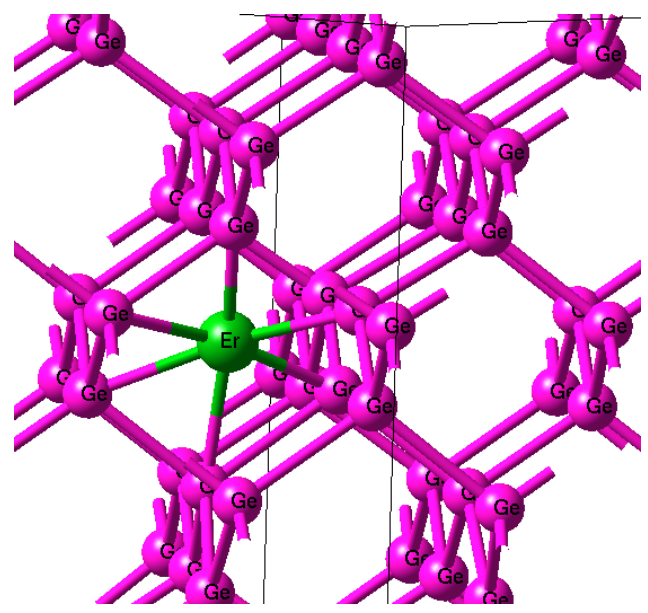

(d)

Fig. 2 Relaxed geometric structures for the $\mathrm{H}$ configuration of RE interstitials in Ge. (a) Ce, (b) Pr, (c) Eu and (d) Er. 


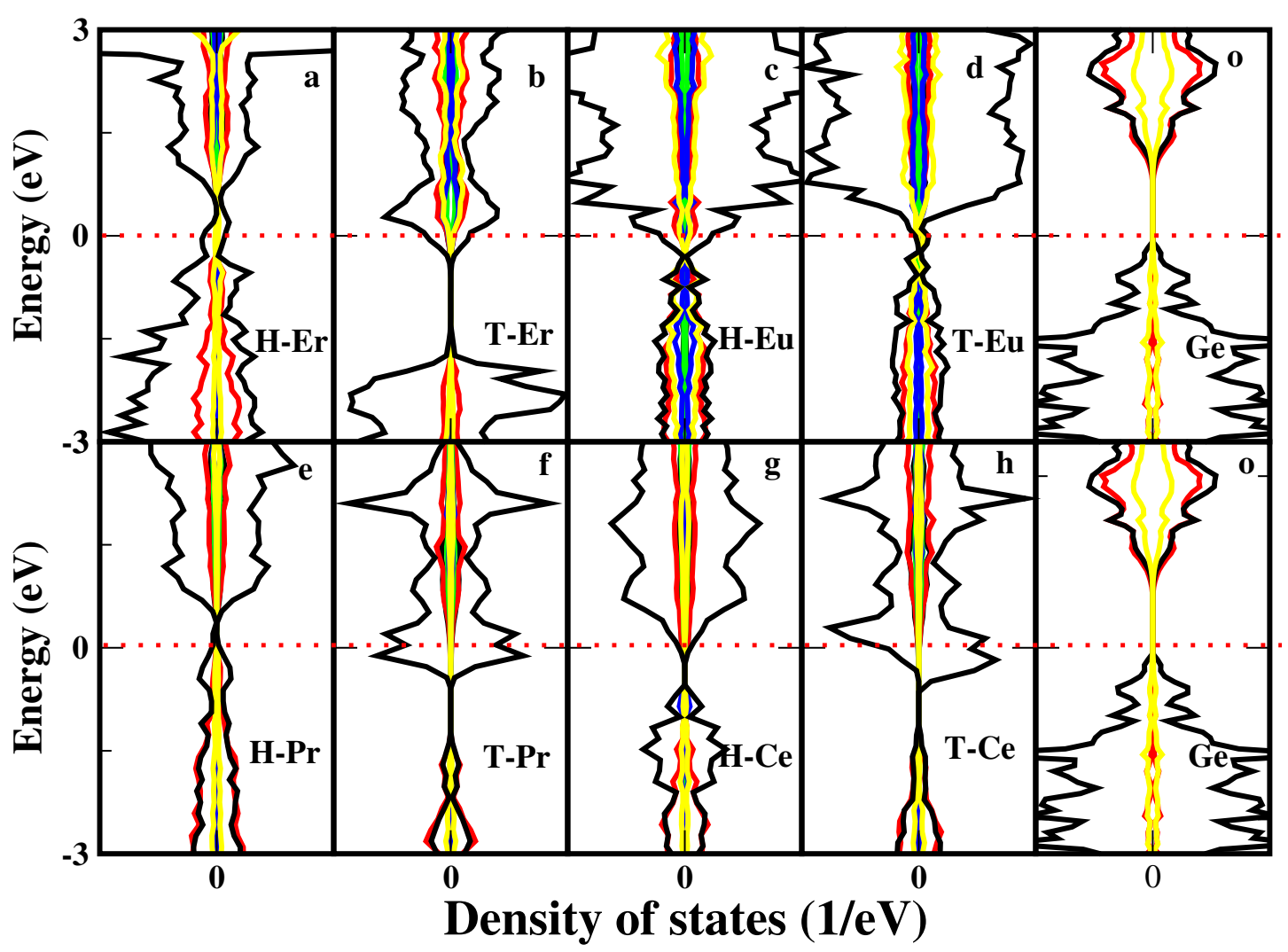

Fig. 3 Plot showing the spin polarised projected density of states (PDOS) of RE interstitials in Ge; (a) H configuration of Er interstitial in Ge (H-Er), (b) T configuration of Er interstitial in Ge (T-Er), (c) H configuration of Eu interstitial in Ge (H-Eu), (d) T configuration of Eu interstitial in Ge (T-Eu), (e) H configuration of Pr interstitial in Ge (H-Pr), (f) T configuration of $\mathrm{Pr}$ interstitial in Ge (T-Pr), (g) H configuration of Ce interstitial in Ge (H-Ce), (h) T configuration of Ce interstitial in Ge (T-Ce) and (o) pristine Ge. The Fermi level (at $\epsilon_{f}=0$ $\mathrm{eV}$ ) is shown by the dashed horizontal line. The majority (spin-up) and minority (spin-down) density of states are shown as right and left halves of each plot. 

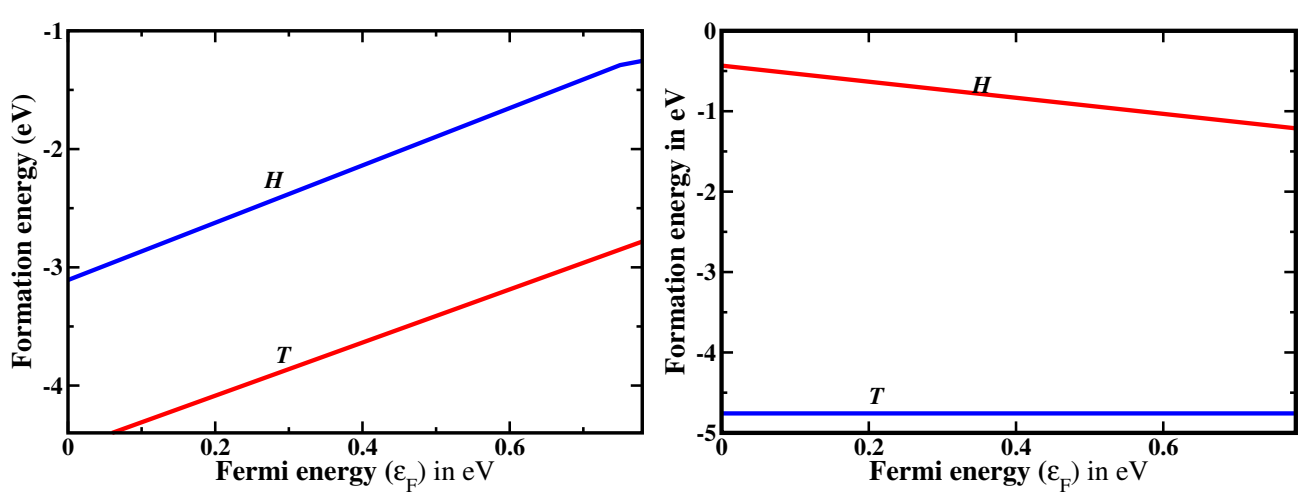

(a) The $\mathrm{H}$ and $\mathrm{T}$ configurations of Ce interstitial (b) The $\mathrm{H}$ and $\mathrm{T}$ configurations of the Pr interstiin Ge, showing donor level for the $\mathrm{H}$ configuration. tial in Ge, there is no charge state transition level

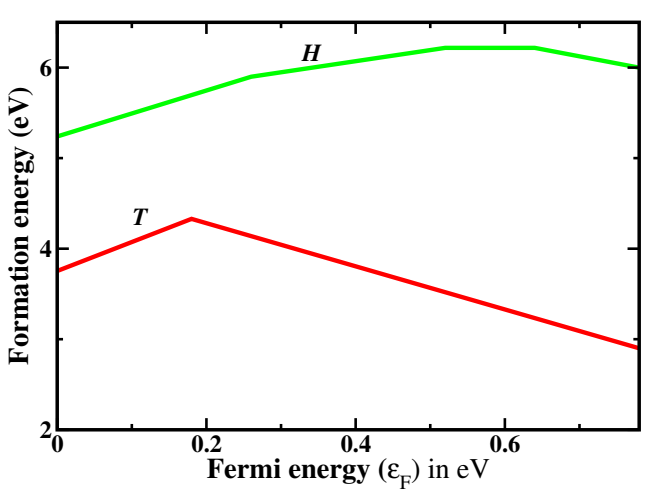
induced by this defect.

(c) The $\mathrm{H}$ and $\mathrm{T}$ configurations of Eu interstitial (d) The $\mathrm{H}$ and $\mathrm{T}$ configurations of Er interstitial

(c) The $\mathrm{H}$ and $\mathrm{T}$ configurations of Eu interstitial (d) The $\mathrm{H}$ and $\mathrm{T}$ configurations of Er interstitial
in Ge, showing negative- $\mathrm{U}$ properties for the $\mathrm{T}$ in Ge. The Er interstitial in Ge displays the propconfiguration.

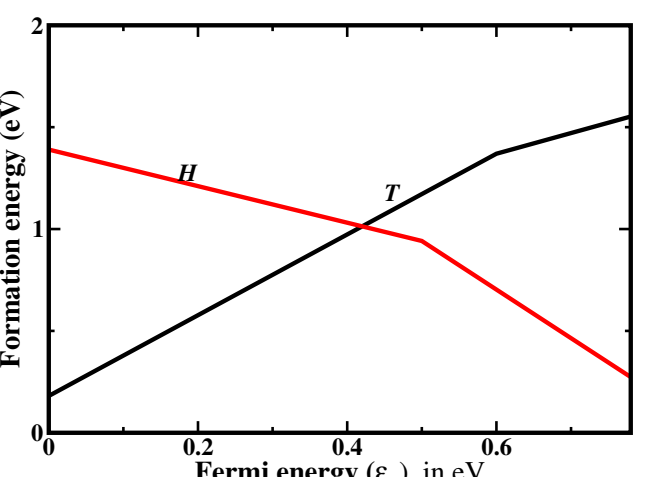
erties of charge state controlled metastability.

Fig. 4 Plot of formation energy as a function of the Fermi energy for the $\mathrm{H}$ (hexagonal) and $\mathrm{T}$ (tetrahedral) configurations of RE interstitials in Ge. 


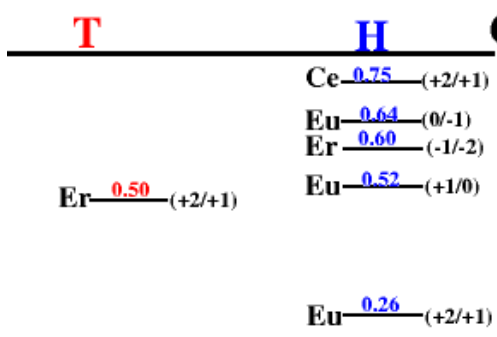

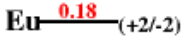

\section{CBM}

$\mathrm{Ce}-2.25-(+2 /+1)$

Eu- $(+2 /+1)$

\section{VBM}

Fig. 5 Calculated negative $-U$, acceptor and donor transition energy levels for both the tetrahedral $(\mathrm{T})$ and hexagonal $(\mathrm{H})$ configurations of RE interstitials in Ge. All energy levels $(\mathrm{eV})$ are with respect to the VBM. 